\title{
Scalable Approach for Content-Based Image Retrieval in Peer-to-Peer Networks
}

\author{
Akshatha M N*, Chaitra S, Monisha B S \\ Department of CSE, GSSSIETW, Mysuru
}

DOI: https://doi.org/10.21467/proceedings.1.27

* Corresponding author email: akshatha.m.n.gowda@gmail.com

\begin{abstract}
Peer-to-peer networking offers a modular solution for sharing visual data across the network. it is a demand issue to perform CBIR in peer-to-peer networks. While most of the existing methods focus on LSH visual features and have limitations of flexibility. In proposed system we use an innovative approach that is a $\mathrm{p} 2 \mathrm{p}$ network with CBIR by employing the BOVW model. As images are scattered across the $\mathrm{p} 2 \mathrm{p}$ network, which makes a static codebook less effective for retrieval tasks. here we propose a codebook updating method by sharpen the mutual information between the follow -up codebook, and the workload balance among peers that manage different code words. In order to further improve retrieval performance and reduce network cost.
\end{abstract}

\section{INTRODUCTION}

Distributed (P2P) systems, which are framed by similarly advantaged hubs interfacing with each other in a self-sorting out way, have been a standout amongst the most critical models for information sharing. Unlike multimedia pages which mainly consist of textual documents such as news, blog articles, multimedia files play a leading role in most P2P networks. The ever-crowding amount of multimedia data and computational power on P2P networks exposes both the need and potential for large scale multimedia. While P2P networks are well known for their novel, and file sharing, providing prolonged search functionality such as content-based image retrieval (CBIR) faces the following challenges:

1) Mainly data in P2P networks is distributed among different nodes, thus a CBIR algorithm needs to evidence and search for images in a shared way;

2) Unlike shared servers/clouds, nodes in P2P networks have limited network bandwidth and computational power, thus the algorithm should keep the network cost low and the load among the nodes should be equal;

To support content indexing and avoid the busy traffic, structured overlay networks such as Distributed Hash Tables (DHTs) are often used at the top of a physical network. When nodes are order in particular way then the message passing between the nodes are flexible, For the CBIR functionality, most of the existing systems adopt a global feature approach: an image is

(C) 2018 Copyright held by the author(s). Published by AIJR Publisher in Proceedings of the $3^{\text {rd }}$ National Conference on Image Processing, Computing, Communication, Networking and Data Analytics (NCICCNDA 2018), April 28, 2018.

This is an open access article under Creative Commons Attribution-NonCommercial 4.0 International (CC BY-NC 4.0) license, which permits any non-commercial use, distribution, adaptation, and reproduction in any medium, as long as the original work is properly cited. ISBN: $978-81-936820-0-5$ 
represented as a high dimensional feature vector (e.g., color histogram), and the relationship between files is scaled using the distance between two feature vectors. On the other hand, the bag-of-visual-words (BoVW) model has been successfully utilized for large scale image retrieval [8]. In the BoVW model, each image is represented with a bag of local features, which mimics the bag-Of-words (BoW) model where each document is a collection of unordered words.2 Generally, to employ the BoVW model, the following three steps are required [9], [10]: Firstly, a number of local regions (through image segmentation or uniform image partitioning) or key points (through key point detection algorithms will be identified from an image and each region or key point will be represented with a high dimensional descriptor.

In our experiments, the widely used Scale-Invariant Feature Transform (SIFT) descriptor [12] is employed. Secondly, since the features extracted are in a continuous space, a codebook is generated to quantize the feature vectors into discrete codeword's, thus an image can be interpreted as a set of features codeword's. One of the most commonly used quantization schemes is nearest-neighbor quantization (e.g., k-means [9], [10]), where each feature vector is represented by its nearest codeword centroid, and the codebook forms a Voronoi partitioning of the feature space. Lastly, similar to the BoW model, statistical distributions of the codeword's in a given image is utilized to represent the image. In this paper we utilize the wellstudied tf-idf weighting scheme and cosine distance as the similarity measurement.

\section{LITERATURE SURVEY}

Image Retrieval in $p 2 p$ Networks: The problem of searching images according to their semantic contents is very challenging since there are many factors affecting the performance like resolution, illumination variations and occluded objects. This motivates the need for an intermediate representation such as visual vocabulary. We have designed a P2P-CBIR system which is based on Bag of Word model and uses the visual vocabulary for image retrieval. This approach begins with the selection of some interest points in an image. These interest points carry properties such as invariance to several image transformations, illumination, etc., then, it defines regions in the locality of these points and extracts the descriptors from them. Each image in a data set is later represented in terms of this visual vocabulary in the form of a model vector.

Visual Feature Extraction: In a bag of word approach, for the representation of the contents of an image (visual features), a set of interest points are chosen, and the features are extracted locally from the nearby area. To select scale invariant interest points, their localization is performed on a Gaussian scale-space. In the proposed P2P-CBIR, the SURF (SpeededUp Robust Features) features are extracted to represent the visual content of an image. These features ensure higher repeatability and distinctiveness. Moreover, the extraction speed of this approach is very fast, when compared with the SIFT features. This approach begins with the localization of interest points. Fast approximation of the Hessian matrix is computed first.

Proceedings of the $3^{\text {rd }}$ National Conference on Image Processing, Computing, Communication, Networking and Data Analytics (NCICCNDA 2018) 
Scalable Approach for Content-Based Image Retrieval in Peer-to-Peer Networks

Then the local maxima of the Hessian matrix determinant are chosen as an interest point. This process is carried out on several octaves of a Gaussian scale-space and the correct scale is automatically selected from the Hessian determinant

BoVW Model: The bag-of-visual-words (BoVW) model represents each image with a bag of quantized codewords derived from local features and measures the similarity between images with the BoVW histogram analogous to a bag-of-words (BoW) model of text retrieval [10]. The retrieval process is typically supported by an inverted index. Though we are not aware of any BoVW based P2P CBIR systems, many existing P2P text retrieval systems build a distributed inverted index in a highly efficient manner over DHT, using term ID as key and document ID as value [16], [17], [18], [19]. Generally, there are two strategies to distribute index tuples: document partition (or local indexing), and term partition (or global indexing), both are well exploited in the literature [31], [32], [33]. With document partition, each node manages an index for a subset of documents. A query will be sent to all index nodes and be answered by combining the lists of candidate documents returned from them. With term partition, each node manages an index for a subset of terms. A query will only be sent to the nodes managing corresponding terms and answered by combining the inverted list returned from them. Therefore, document partition typically has a higher network cost than term partition, especially when the index has a good term sparsity [32]. This is not a very big issue in shared-memory or distributed servers, but does pose a challenge in P2P networks, as the nodes in P2P networks are loosely coupled and have much lower bandwidth. As a result, term partition is a more popular choice in P2P networks [16], [17], [18], [19]. To further reduce the network cost and tackle the issue of workload balance with term partition, different techniques have been proposed. For BoVW based CBIR in P2P networks, [34] proposes a codebook resampling mechanism to split the overloaded codewords and merge the under loaded codewords to maintain a balanced workload among different codewords. However, it does not take the relevance information into account. In addition, the split/merge is based on random re-sampling, which is heuristic.

\section{SYSTEM ARCHITECTURE}

Peep-to-Peer network is a newly emerging paradigm of distributed computing. In P2P architecture, files are stored at the end user machines (peers) rather than on a central server and transferred directly between peers. Distributed architecture for CBIR has many advantages over a conventional client server-based image retrieval systems, such as comprehensiveness of information, better scalability and reliability. The detailed architecture of a proposed P2P-CBIR system is given in the next section which is based on a Gnutella network. As every query is broadcast to every peer under the Gnutella protocol, each peer has to waste resources in processing irrelevant queries. This query message flooding within the network increases the traffic. We tackle this problem with a proposed peer clustering approach 
which groups the peers that share similar image data that helps to search the images more efficiently in a P2P network.

\section{Image Sharing \& Retrieval in P2P-CBI}

We briefly outline the process of retrieval of images in the P2P-CBIR system. First, each peer performs feature extraction on its shared collection of images using the client program. A client program uses a Bag of Word approach for feature extraction. With the help of this client program, each peer keeps its local index of the feature vectors of its image collection. When a peer, triggers a retrieval process by providing a query image, it extracts the features of the query image and send back the feature vector, enclosed in a query message, to all its connected peers. Subsequently, other peers compare this query with their feature vector index and find the similar images with the help of similarity measure. Finally, they return results back to the requester. Further, these peers propagate the query to their connected peers and this process is repeated until it covers the largest portion of a network. The proposed P2P-CBIR system operates on the Gnutella network. To support the image query functionalities, two types of query messages are used. Image_Query is a special type of query message which contains the information about the feature extraction method and the feature vector of the query image. Query_Hit message responds to the Image_Query message. It carries information such as, the location, name of file and size of the retrieved images, and their similarity measure. Below figure depicts the architecture of the proposed P2P-CBIR system.

\section{USER INTERFACE}

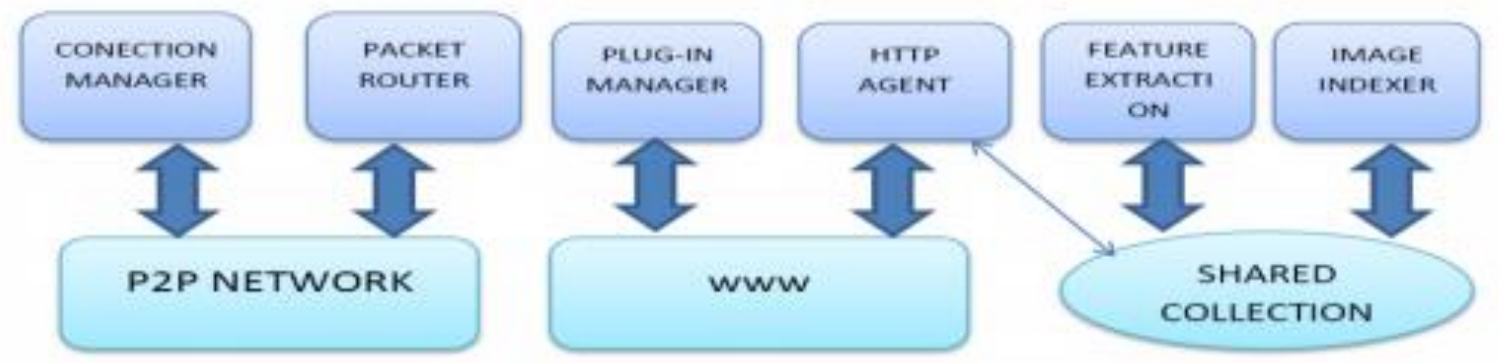

Connection Manage is responsible for setting up and to manage the TCP connection between P2P-CBIR clients. Packet Router controls the routing, assemble and disassemble messages within the network and between the various components of the client program. Plug-in Manager manages the download and storage of different feature extraction plug-ins and their interaction with a feature extractor module and Image Indexer. HTTP Agent is a small webserver that is accountable for complete file download requests from other P2P-CBIR peers

Proceedings of the $3^{\text {rd }}$ National Conference on Image Processing, Computing, Communication, Networking and Data Analytics (NCICCNDA 2018) 
Scalable Approach for Content-Based Image Retrieval in Peer-to-Peer Networks

using the HTTP protocol. Feature Extractor works together with the Plug-in Manager to carry out feature extraction and thumbnail generation from the shared image collection.

\section{IMPLEMENTATION}

The process of image retrieval in a P2P environment is summarized in following steps:

\subsection{Preprocessing}

The Feature Extractor module extracts features and generates thumbnails for all shared images using a feature extraction method. The Image Indexer module then indexes the image collection using the extracted feature vectors. Sharing the load of this computationally expensive task among peers by allowing them to store and index their image collection helps to tackle the bottleneck problem.

\subsection{Connection Establishment}

The peer joins the P2P network using this module. The Connection Manager module requests the Bootstrap Server, which is program that stores and answers a list of peers currently connected to the network. After knowing the IP address, the peer connects to the P2P network by connecting to currently available peers.

\subsection{Query Message Routing}

When a peer initiates a process of retrieval by giving a query to search for similar images, the Feature Extractor module processes the query image and assembles the Image_Query message. Similarly, when other peers receive the Image_Query messages, they carry out two operations: Query Message Propagation and Local Index Look Up. Once similar images are retrieved, the peer returns the Query_Hit back to the requester through Packet Router module.

\subsection{Display of Query Result}

When an Image Query_Hit message returns to the requester, users will get a list of the locations and size of the images which are matched to the query. To retrieve the query result, the HTTP Agent downloads the thumbnails that are generated during the preprocessing stage, or an entire image from the peer using HTTP protocol.

\section{CONCLUSION}

We present a bag-of-visual-words (BoVW) model-based approach for content based image retrieval (CBIR) in peer-to-peer (P2P) networks. In order to overcome the difficulty in generating and maintaining a global codebook when the BoVW model is deployed in P2P networks, we formulate the problem of updating an existing codebook as optimizing the retrieval accuracy and workload balance. As a result, the proposed approach is scalable to the number of images shared within a P2P network and the evolving nature of P2P networks. In order to further improve the retrieval performance of the proposed approach and reduce network cost, indexing pruning techniques are applied. 


\section{References}

[5] M. Steiner, T. En-Najjary, and E. W. Biersack, "Long term study of peer behavior in the KAD DHT," IEEE/ACM Transactions on Networking, vol. 17, no. 5, pp. 1371-1384, Oct. 2009.

[6] H. Schulze and K. Mochalski, “Internet study 2008/2009,” Internet Studies, ipoque, 2009.

[7] I. Stoica, R. Morris, D. Karger, M. F. Kaashoek, and H. Balakrishnan, "Chord: A scalable peer-to-peer lookup service for internet applications," in ACM Conference on Applications, Technologies, Architectures, and Protocols for Computer Communications, 2001, pp. 149-160.

[8] S. Ratnasamy, P. Francis, M. Handley, R. Karp, and S. Shenker, "A scalable content-addressable network," in ACM Conference on Applications, Technologies, Architectures, and Protocols for Computer Communications, 2001, pp. 161-172.

[9] M. Mordacchini, L. Ricci, L. Ferrucci, M. Albano, and R. Baraglia, "Hivory: Range queries on hierarchical voronoi overlays," in IEEE International Conference on Peer-to-Peer Computing, Aug. 2010, pp. 1-10.

[10] Y. Tang, S. Zhou, and J. Xu, "LIGHT: A query-efficient yet lowmaintenance indexing scheme over DHTs," IEEE Transactions on Knowledge and Data Engineering, vol. 22, no. 1, pp. 59-75, Jan. 2010.

[11] L. Zhang, Z. Wang, and D. Feng, "Efficient high-dimensional retrieval in structured P2P networks," in IEEE International Conferenceon Multimedia and Expo Workshops, Jul. 2010, pp. 1439-1444.

[12] H. J'egou, M. Douze, and C. Schmid, "Improving bag-of-features for large scale image search," International Journal of Computer Vision, vol. 87, pp. 316-336, 2010.

[13] J. Sivic and A. Zisserman, "Video Google: A text retrieval approachto object matching in videos," in IEEE International Conference on Computer Vision, vol. 2, 2003, pp. 1470-1477.

[14] J. Yang, Y.-G. Jiang, A. G. Hauptmann, and C.-W. Ngo, "Evaluating bag-of-visual-words representations in scene classification," in ACM International Workshop on Multimedia Information Retrieval, 2007, pp. 197-206.

[15] K. Mikolajczyk and C. Schmid, "An affine invariant interest point detector," in European Conference on Computer Vision, 2002, pp. 128-142.

[16] D. G. Lowe, "Object recognition from local scale-invariant features," in IEEE International Conference on Computer Vision, vol. 2, 1999, pp. 1150-1157.

[17] S. Rhea, D. Geels, T. Roscoe, and J. Kubiatowicz, "Handling churnin a DHT," in USENIX Annual Technical Conference. Boston, MA,USA, 2004, pp. 127-140.

[18] D. Belson, "The state of the internet, 2nd quarter, 2013 report,"Akamai, 2013.

[19] Y. Rui, T. S. Huang, M. Ortega, and S. Mehrotra, "Relevance feedback: A power tool for interactive contentbased image retrieval," IEEE Transactions on Circuits and Systems for Video Technology, vol. 8, no. 5, pp. 644655, Sep. 1998.

[20] C. Tang, Z. Xu, and S. Dwarkadas, "Peer-to-peer information retrieval using self-organizing semantic overlay networks," in ACM Conference on Applications, Technologies, Architectures, and Protocols for Computer Communications, 2003, pp. 175-186.

[21] P. Reynolds and A. Vahdat, "Efficient peer-to-peer keyword searching," in ACM/IFIP/USENIX International Conference on Middleware, 2003, pp. 21-40.

[22] Q. Xu, H. T. Shen, Y. Dai, B. Cui, and X. Zhou, "Achieving effective multi-term queries for fast DHT information retrieval," in International Conference on Web Information Systems Engineering (WISE), 2008, pp. 20-35.

[23] H. Chen, J. Yan, H. Jin, Y. Liu, and L. M. Ni, "TSS: Efficient term set search in large peer-to-peer textual collections," IEEE Transactions on Computers, vol. 59, no. 7, pp. 969-980, 2010.

[24] M. Batko, D. Novak, F. Falchi, and P. Zezula, "Scalability comparison of peer-to-peer similarity search structures,” Future Generation Computer Systems, vol. 24, no. 8, pp. 834 - 848, 2008. 